\title{
Math goes history
}

\author{
Nataša Djurdjevac Conrad, Christof Schütte \\ und Rupert Klein
}

\begin{abstract}
Menschliche Gesellschaften haben seit jeher mit gravierenden Veränderungen ihrer Lebensbedingungen zu kämpfen, sei es durch steigende Bevölkerungsdichte, Ressourcenknappheit, klimatischen Wandel oder Naturkatastrophen. Das Verständnis der zugrundeliegenden komplexen Prozesse in historischen Zivilisationen ist ein Hauptanliegen der Zusammenarbeit zwischen $\mathrm{MATH}^{+}$ und der reichen Berliner Wissenschaftslandschaft in der Antikenforschung.
\end{abstract}

Eine zentrale Herausforderung hierbei ist die Konstruktion plausibler, evidenzbasierter Narrative für die komplexen Veränderungsprozesse, die vergangene Gesellschaften beeinflussten und prägten. Archäologische Nachweise hierzu stammen aus verschiedenen Quellen wie Ausgrabungen oder Aufzeichnungen von Klima- und anderen Umweltfaktoren. Solche Nachweise sind komplex: Sie sind oft fragmentarisch und waren vor ihrer Aufzeichnung vielfältigen Transformationen unterworfen, die Erhaltungsgrad, Fundort und -umgebung beeinflussen. Aufgrund der inhärenten Schwierigkeiten, aus den Daten die zugrundeliegenden räumlich-zeitlichen Prozesse abzuleiten, ist ihre Interpretation mittels fortgeschrittener mathematischer Modellierung und formalisierter Argumentation eine attraktive Alternative.

$\mathrm{MATH}^{+}$zielt darauf ab, wichtige wissenschaftliche Konzepte für die Beschreibung von gesellschaftlichen Transformationsprozessen wie zum Beispiel „Resilienz", „Vulnerabilität“ oder „Migrationswellen“ in mathematischer Form darzustellen. Es sollen Modellentwicklungen für historische Veränderungsprozesse wie die Ausbreitung von Innovationen in alten Kulturen vorangetrieben und im Lichte der entwickelten Veränderungskonzepte analysiert werden. Und es gilt, relevante Forschungsdaten mit fortgeschrittenen Techniken der Datenanalyse zu charakterisieren sowie Methoden für die Integration der Daten in die in Entwicklung befindlichen Modelle bereitzustellen. Die mathematischen Modelle, die zum Einsatz kommen, sind sehr vielfältig. Sie reichen von der Kategorientheorie zur Konzeptionalisierung von Grundbegriffen über stochastische agentenbasierte Modelle zur Beschreibung der Bedeutung zwischenmenschlicher Interaktion in der Innovationsausbreitung bis zur Analyse von Trajektorien auf Riemannschen Form-Mannigfaltigkeiten zur Einordnung sich zeitlich verändernder Formgebung von Gebrauchsgütern, Bau- und Kunstobjekten.

Die geplanten Forschungsaktivitäten sind nicht auf den Austausch mit den Antikenwissenschaften beschränkt. Ganz im Gegenteil sollen die durch mathematische Abstraktion gewonnenen Methoden auch in anderen Feldern der Geistes- und Sozialwissenschaften zum Einsatz kommen, wie etwa bei der Erforschung sozialer Dynamik heutiger gesellschaftlicher Transformationsprozesse oder in der Nach-
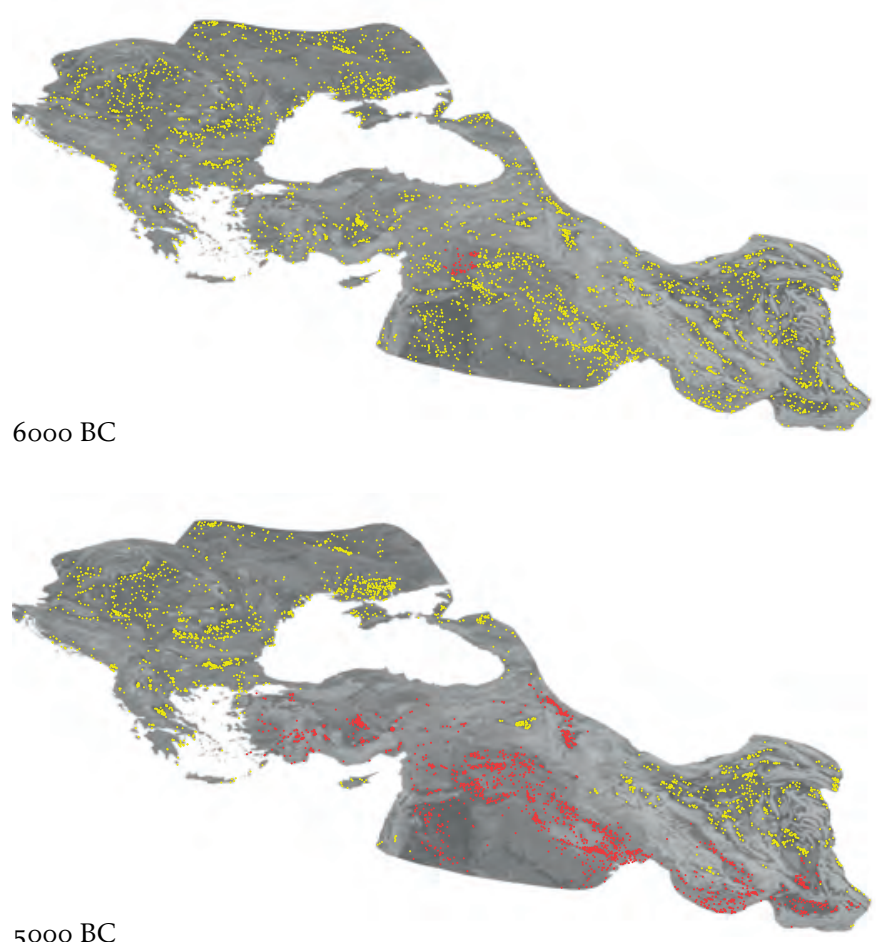

$5000 \mathrm{BC}$

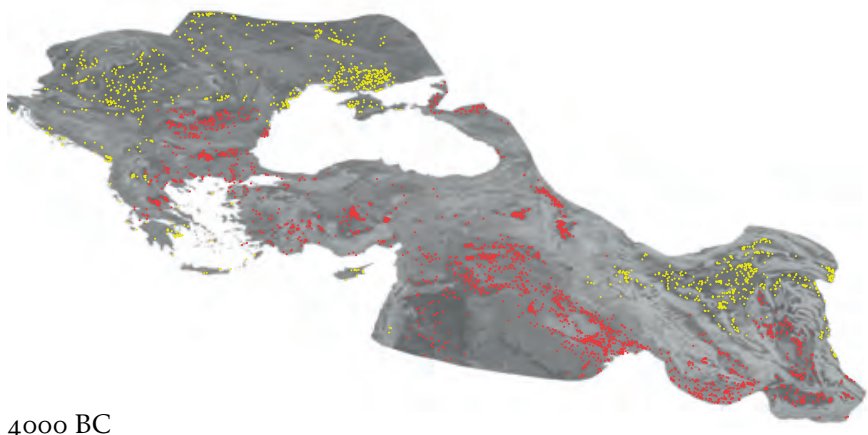

Die Abbildung zeigt den Fortschritt der Ausbreitung des Wollschafs und der damit einhergehenden Wollverarbeitungstechnologie im vorderen Orient und dem Balkan zwischen 6000 und 4000 v. Chr. Es resultiert aus der Simulation eines datengetriebenen agentenbasierten Modells, in dem jeder Punkt eine Gruppe von Hirten repräsentiert, wobei die rote Farbe Hirtengruppen mit Kenntnis der Technologie und gelb solche ohne diese Kenntnis zeigt [Dj.Conrad et.al., EPJ DataSci, 2018]. 
haltigkeitsforschung Dort werden ähnliche Fragestellungen nach den Ursachen oder der Beeinflussbarkeit von Veränderungsprozessen im Umfeld von Energiewende und Klima- wandel diskutiert. Auch hier plant $\mathrm{MATH}^{+}$enge Kooperationen mit exzellenten Partnern aus den entsprechenden Bereichen.

\author{
Dr. Nataša Djurdjevac Conrad, Prof. Dr. Christof Schütte \\ Zuse-Institut Berlin (ZIB), Takustraße 7, 14195 Berlin \\ natasa.conrad@zib.de,schuette@zib.de \\ Prof. Dr. Rupert Klein \\ Freie Universität Berlin, Institut für Mathematik, \\ Arnimallee 6, 14195 Berlin \\ rupert.klein@math.fu-berlin.de
}

\begin{abstract}
Nataša Djurdjevac Conrad leitet die Arbeitsgruppe „Computational Humanities“ am Zuse-Institut Berlin (ZIB) und ist Projektleiterin in MATH ${ }^{+}$. Sie forscht im Rahmen des $M A T H^{+}$Emerging Fields 5 - Concepts of Change in Historical Processes.

Christof Schütte ist Professor für Scientific Computing an der FU Berlin und Präsident des Zuse-Instituts Berlin, wo er den Forschungsbereich "Mathematik für Lebens- und Materialwissenschaften“ leitet. Er ist einer der beiden stellvertretenden Sprecher von MATH ${ }^{+}$.
\end{abstract}

Rupert Klein ist Professor für Scientific Computing an der FU Berlin und Leiter der Arbeitsgruppe „Geophysical Fluid Dynamics“ am Fachbereich Mathematik. Er ist Mitglied des Vorstands und einer der 25 PIs von MATH ${ }^{+}$.

\title{
Tropische Geometrie, lineare Optimierung und Netzwerke
}

\author{
Michael Joswig
}

Das erste Thematic Einstein Semester von $\mathrm{MATH}^{+}$wird im Sommer 2019 stattfinden und den Titel „Network games, tropical geometry and quantum communication" tragen. In diesem Rahmen sind speziell konzipierte Vorlesungen geplant, die kombiniert werden mit Projekten, Vorträgen und Workshops. Höhepunkt des Semesters ist eine internationale Tagung, die vom 3. bis zum 9. Juni 2019 in Berlin stattfinden wird. Das Ziel ist es, drei für sich bereits interessante und umfangreiche Gebiete thematisch zu bündeln und Schnittstellen auszuloten. Die interessanten Anknüpfungspunkte zwischen Quantenkommunikation und Graphenthorie sollen hier nicht vertieft werden. Statt dessen beschränkt sich dieser Text darauf, exemplarisch zu motivieren, wie man von der Geometrie zu Graphen und Netzwerken kommt.

Bereits 2009 schrieben David Speyer und Bernd Sturmfels [10] "... the tropical approach in mathematics ... [is] now an integral part of geometric combinatorics and algebraic geometry. It has also expanded into mathematical physics, number theory, symplectic geometry, computational biology, and beyond." Angefangen hat diese Erfolgsgeschichte mit einem Zählproblem, das in Maxim Kontsevichs Arbeiten zu Gromov-Witten-Invarianten symplektischer Mannigfaltigkeiten auftritt [6]. Grigory Mikhalkin hatte erkannt, dass sich die komplexen algebraischen Kurven im ursprünglichen Problem durch tropische Kurven ersetzen lassen [8].
Letztere haben eine sehr viel einfachere Struktur, enthalten aber immer noch die wesentliche Information. Hannah Markwig hat hierüber schon einmal in den Mitteilungen berichtet [7]. Was ist der Trick?

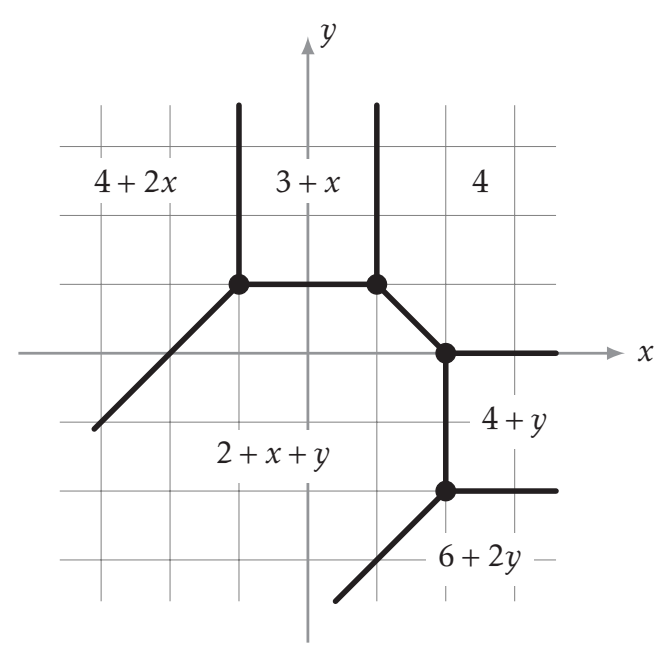

Abbildung 1. Ebene tropische Quadrik zu $F(x, y)$ in (2). Jede Region ist markiert durch denjenigen Term, an dem das Minimum angenommen wird. Die tropische Kurve liegt quasi „dazwischen“, also dort wo dieses Minimum mehrfach angenommen wird. 